\title{
High Definition Spectral Domain Optical Coherence Tomography Findings in Three Patients with Solar Retinopathy and Review of the Literature
}

\author{
Kevin C. Chen ${ }^{1}$, Jesse J. Jung ${ }^{1}$ and Alexander Aizman ${ }^{*}, 1,2$ \\ ${ }^{I}$ Department of Ophthalmology, New York University School of Medicine, New York, NY, USA \\ ${ }^{2}$ Einhorn Clinical Research Center, The New York Eye and Ear Infirmary, New York, NY, USA
}

\begin{abstract}
Purpose: To describe ocular findings in 3 cases of solar retinopathy using high definition, spectral domain optical coherence tomography (SD-OCT) and review the literature for optical coherence tomography (OCT) characteristics associated with worse vision.

Methods: Case series and retrospective review of clinical features and Spectralis SD-OCT (Heidelberg Engineering, Vista, California, United States of America). A literature review of OCT findings in cases of solar retinopathy reported on MEDLINE was also performed and analyzed.

Results: Six eyes of 3 patients with solar retinopathy revealed significant foveal pathology. Visual acuity ranged from Snellen 20/30 to 20/50. High definition SD-OCT demonstrated defects at the level of the inner and outer segment junction of the photoreceptors as well as in the inner high reflective layer. There was a significant correlation between chronic disruption of the inner photoreceptor junction with worse vision based on the current case series and literature review.

Conclusions: Screening patients with exposure to central foveal damage from solar retinopathy with high definition SDOCT improves diagnosis and assessment of photoreceptor damage and vision loss.
\end{abstract}

Keywords: Solar retinopathy, fovea, spectral domain optical coherence tomography, Spectralis SD-OCT.

\section{INTRODUCTION}

Solar retinopathy from prolonged exposure to solar radiation is a rare but well-recognized clinical entity of vision loss and macular damage [1]. Exposure to arc welding, viewing of a solar eclipse or direct sun-gazing in religious ritual participants, military personnel, people with mental disturbances and sunbathers has been identified as direct causes of solar retinopathy [2]. Symptoms typically are bilateral but may be asymmetric and include blurred vision, a central or paracentral scotoma, chromatopsia, metamorphopsia, photophobia and headache [3]. Fundus exam reveals central foveal changes with a small yellowishwhite spot and surrounding grey, granular pigmentation [4]. Over time, the spot may evolve and appear as a sharply circumscribed red spot at the fovea [1]. Histopathological studies have demonstrated that both the retinal pigment epithelial (RPE) layer [5] and outer segments of the photoreceptor layer are most susceptible to solar damage [6]. It is unclear which effect occurs first, but it is hypothesized that the melanin in the RPE layer absorbs the extent of the light energy subsequently damaging these epithelial cells and surrounding photoreceptors $[4,7]$.

With the innovation of OCT, clinical and histopathological studies have been correlated to findings

*Address correspondence to this author at the Department of Ophthalmology, New York University School of Medicine, 4050 Nostrand Ave, Suite M1 Brooklyn, NY 11235, USA; Tel: 718-646-2025;

Fax: 718-646-2024; Email: alaizman@aol.com seen on the OCT. Previous reports utilizing the time domain OCT developed by Stratus (Carl Zeiss Meditec, Inc., Dublin, California, USA) and early platforms of time domain Zeiss OCT-3 (Zeiss Humphrey Instruments, Dublin, California) have shown defects either in the photoreceptor inner and outer segments $[4,8-22]$ or the RPE layer [3,7-11,21,22]. The following case series utilized high definition, SD-OCT imaging from the Spectralis (Heidelberg Engineering, Vista, California, USA) to attempt to characterize the exact layer of foveal pathology seen in solar retinopathy. A review of the literature comparing OCT findings and solar retinopathy was also performed to analyze any relationships between visual acuity and OCT parameters.

\section{METHODS}

A retrospective chart review of 6 eyes from 3 patients who were referred to the medical retinal consultation service of one retinal specialist were identified as having decreased visual acuity secondary to solar retinopathy. Each patient had a history of solar exposure and underwent comprehensive ocular examinations including best-corrected visual acuity (BCVA), anterior segment examination, dilated biomicroscopic examination of the macula and indirect ophthalmoscopic evaluation of the retinal periphery. Additional evaluation included Spectralis SD-OCT (Heidelberg Engineering, Vista, California, United States of America) analysis of the macula evaluating for any areas of retinal disruption or atrophy.

A review of the literature was also performed including all cases of solar retinopathy with optical coherence 
tomography reported on MEDLINE and cited in the previous reported cases. Inclusion criteria were reports that included OCT findings, definitive history of solar exposure, reported time from exposure to examination and published articles that were interpretable in English. Review included age; gender; time period from exposure to examination; BCVA in eye examined without another confounding diagnosis for vision changes; funduscopic macular findings; and OCT findings as described by the respective authors, which included disruption of outer photoreceptors, inner photoreceptors, inner high reflective layer and RPE layers. The review included published literature up to October 2011.

Data from our case series and the literature were evaluated to determine if any OCT parameters were related to loss of vision. Statistical analysis was performed with Microsoft Excel (Microsoft Corporation, United States). Odds ratios were used to compare time of exposure to examination with RPE and photoreceptor lesions and were evaluated with 95\% confidence intervals and Fisher's exact test. Pearson's correlation was used to analyze the correlation between age and $\log$ MAR visual acuity as well as central foveal thickness and logMAR visual acuity. MannWhitney $U$ test was used to correlate time of exposure to examination; gender, RPE defects, inner high reflective layer lesions, outer segment lesions and inner segment lesions with $\log$ MAR visual acuity. An exploratory p-value of 0.05 was used as the level of statistical significance.

\section{RESULTS}

\section{Case 1}

An 11 year old Filipino boy was referred for retinal evaluation after complaining of blurry vision in both eyes. He reported looking at the sun for a few minutes while his parents participated in an outdoor religious ceremony one week prior to presentation. BCVA was 20/50 (logMAR $0.398)$ in the right eye and 20/40 (logMAR 0.301) in the left eye. Color vision was intact by Ishihara plate testing and Amsler grid and intraocular pressures were within normal limits. The anterior segment appeared normal. On funduscopic exam, possible macular hole defects in the outer retinal layer as well as abnormal foveal light reflexes were noted in both eyes.

High definition SD-OCT showed focal excavation of the inner high reflective layer and the inner and outer segment junction of the photoreceptors (Fig. 1). The external limiting membrane was hyperreflective just anterior to the excavation. The RPE and vitreoretinal interfaces were
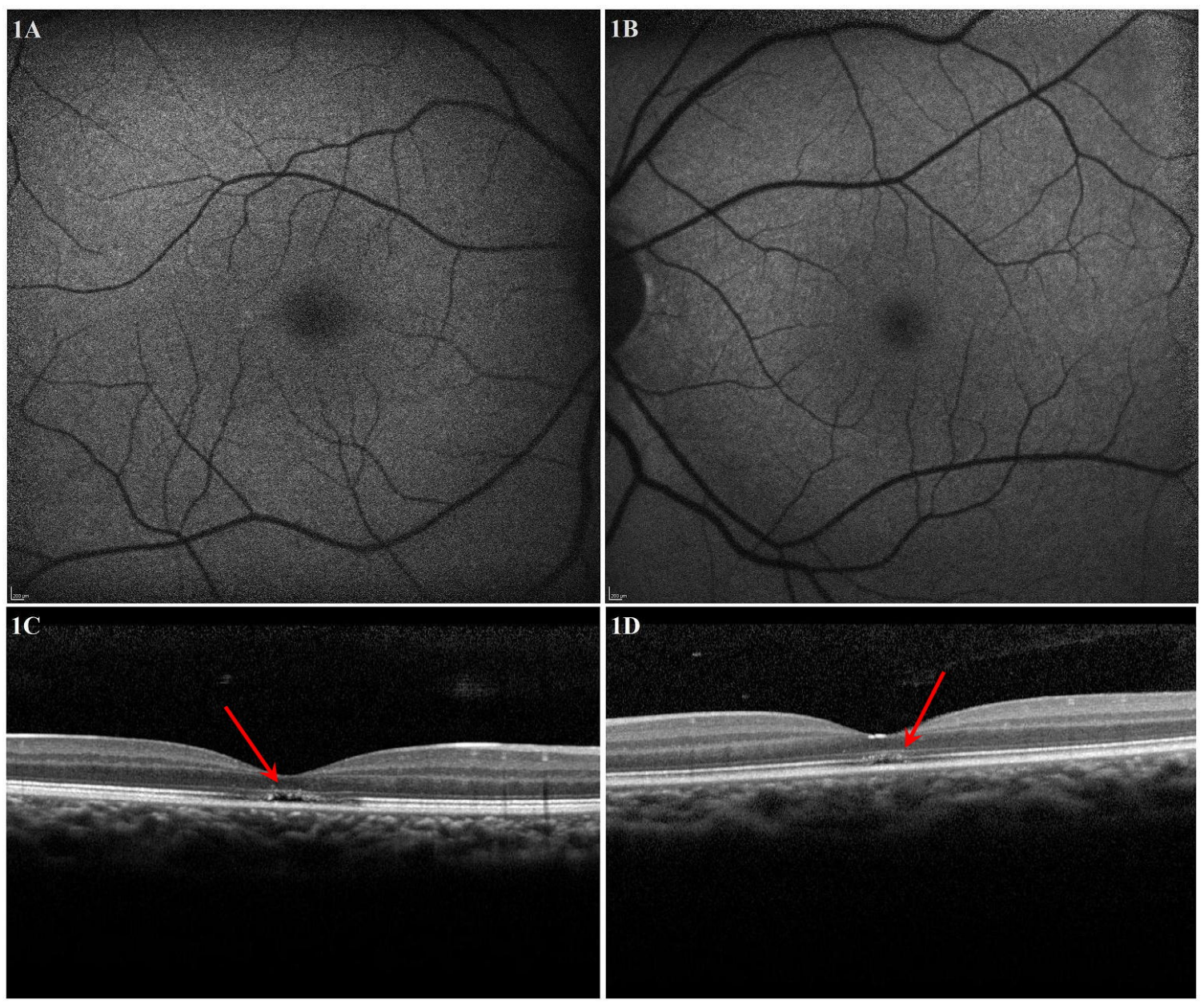

Fig. (1). Case 1: (A) Right eye macula autofluorescence Optical Coherence Tomography (OCT) image and (B) left eye macula autofluorescence OCT image showing no autofluorescent abnormalities. (C) OCT through the right eye fovea showing defects in the inner segment, outer segment and inner high reflective layers. The external limiting membrane is hyperreflective anterior to the foveal defect. (D) OCT through the left eye fovea showing similar defects in the inner segment, outer segment, and inner high reflective layers. 
normal in both eyes. Central foveal thickness was 174 microns in the right eye and 187 microns in the left eye.

\section{Case 2}

A 52 year old African American man presented with decreased vision in both eyes. He reported looking at the sun one week prior to presentation and noticed worsening vision. His BCVA was 20/30 (logMAR 0.176) in both eyes. Color vision was intact by Ishihara plate testing and intraocular pressures were normal. Amsler grid was significant for metamorphopsia in both eyes around the point of focus. Anterior segment was unremarkable. Dilated funduscopic exam showed RPE changes and an abnormal foveal light reflex in both eyes.

High definition SD-OCT of the right eye showed a focal excavation of the inner high reflective layer and the inner and outer photoreceptor segment junction (Fig. 2). Mild hyperfluorescence was noted in the external limiting membrane anterior to the lesion. Vitreoretinal interface and RPE were normal in the right eye. SD-OCT of the left eye showed a pigment epithelial detachment parafoveally with no subretinal fluid and a blurring of the inner high reflective layer and a defect at the inner and outer segment junction at the fovea. Central foveal thickness was 218 microns in the right eye and 231 microns in the left eye.

\section{Case 3}

A 23 year old Russian man was referred by his general ophthalmologist to rule out solar retinopathy. He gave a history of sun gazing the week prior. His medical history was significant for bipolar disorder for which he took Depakote and Zyprexa. BCVA was 20/30 (logMAR 0.176) in the right eye and 20/30 (logMAR 0.176) in the left eye. Color vision was intact by Ishihara plate testing and intraocular pressures were normal in both eyes. Amsler grid showed metamorphopsia in the right eye. The anterior segment was unremarkable. Dilated funduscopic exam found possible macular holes and blunting of the foveal reflex in both eyes. High Definition SD-OCT was not performed at this time.

On follow-up one year later, his best corrected vision was 20/25 (logMAR 0.097) in the right eye and 20/30 (logMAR
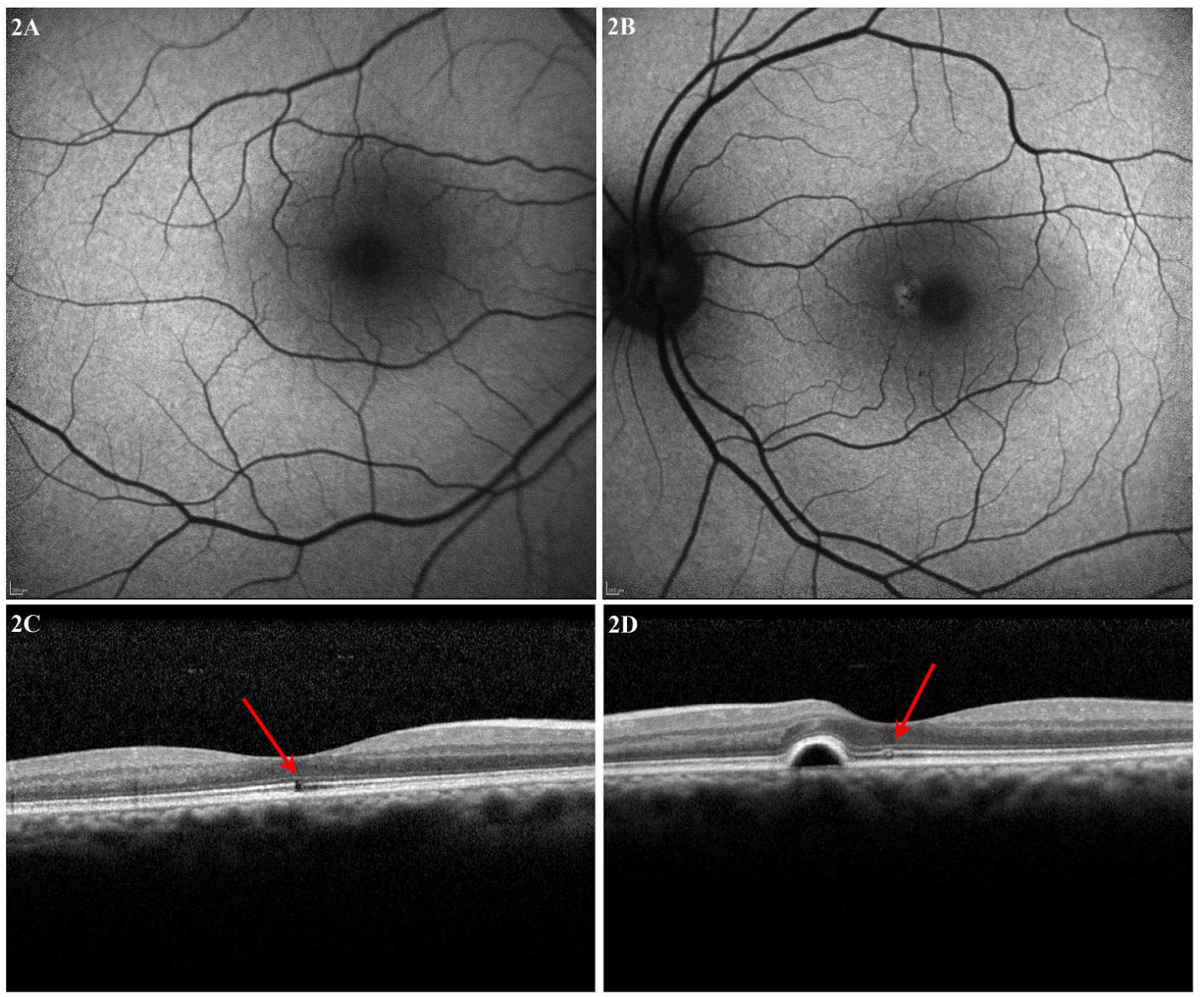

Fig. (2). Case 2: (A) Right eye macula autofluorescence OCT image showing no autofluorescent abnormalities. (B) Left eye macula autofluorescence OCT image with hyperfluorescence parafoveally. (C) OCT through the right eye fovea showing defects in the inner segment, outer segment, and inner high reflective layers. Mild hyperreflectivity is noted in the external limiting membrane anterior to the lesion. (D) OCT through the left eye fovea shows a pigment epithelial detachment parafoveally with no subretinal fluid and disruption of the inner high reflective layer and the inner and outer segment junction. 

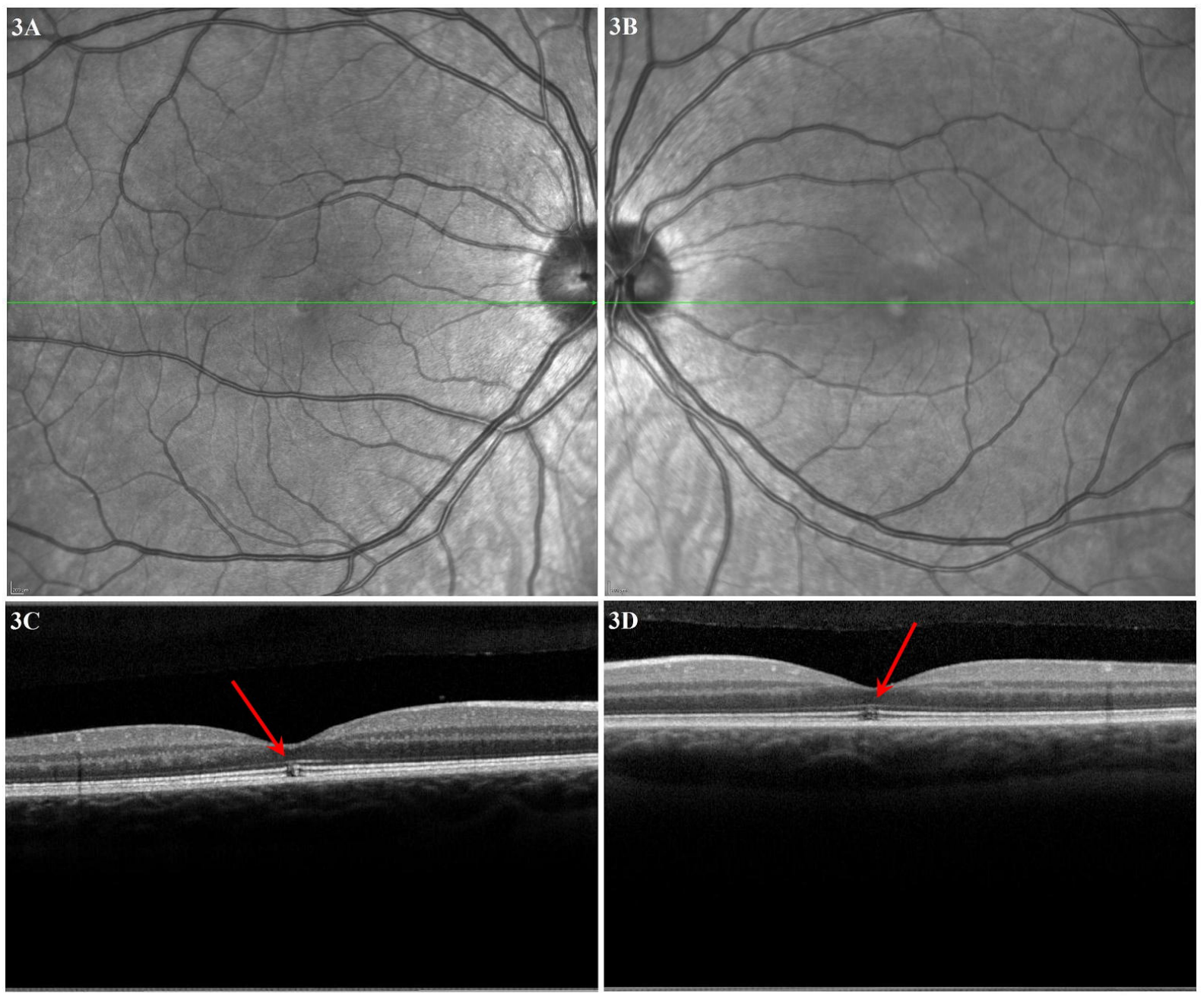

Fig. (3). Case 3: (A) Right eye macula red-free OCT image and (B) left eye macula red-free OCT image showing a brighter foveal area. (C) OCT through the right eye fovea showing lesions in the inner segment, outer segment, and inner high reflective layers. Note the hyperreflective external limiting membrane anterior to the lesion. (D) OCT through the left eye fovea showing disruption in the inner segment, outer segment, and inner high reflective layers.

0.176) in the left eye. High definition SD-OCT showed lesions in the inner high reflective layer extending to the inner and outer photoreceptor segment junction in both eyes (Fig. 3). A hyperfluorescent external limiting membrane was observed anterior to the lesion. The vitreoretinal interface and the RPE were normal in both eyes. Central foveal thickness was 200 microns in the right eye and 204 microns in the left eye.

\section{Literature Review}

Utilizing a MEDLINE search of the literature, 25 papers of solar retinopathy with OCT analysis from 1999 to October 2011 were reviewed. Of the original 25 papers, 21 papers fit the inclusion criteria. A total of 101 eyes from 68 patients (41 males) including eyes from this current case series were analyzed. The average age of the patients was 39.5 years (range 10-86 years). Average Snellen visual acuity was $20 / 61.5$ with range of $20 / 20$ to $20 / 200$ (mean logMAR 0.488 , range $0-1$ ) and median was 20/40 (logMAR 0.301). Mean time between sun exposure and ophthalmic exam was 1999 days (range 1-7300 days) (Table 1).
All 101 eyes were analyzed with OCT.RPE defects were seen in 27 eyes $(26.7 \%)$, inner high reflective layer defects in 67 eyes $(66.3 \%)$, outer segment defects in 67 eyes $(66.3 \%)$ and inner segment defects in 33 eyes (32.7\%) (Table 1).

Eighty-nine eyes had documented time period from exposure. Thirty-seven eyes including the 4 eyes from Case 1 and 2 in this present series were categorized in the acute sun exposure group, defined as within one year from exposure to exam; 47 eyes including the 2 eyes from Case 3 were categorized as chronic sun exposure. The median BCVA was 20/30 (logMAR 0.176) in the acute exposure group and 20/60 (logMAR 0.477) in the chronic exposure group, which differed significantly ( $p<0.001)$ (MannWhitney $U$ test). When compared, the odds ratio of retinal pigment epithelial defects in the acute versus chronic sun exposure groups was clinically significant at $2.78(95 \%$ Confidence Interval (CI) $1.08-7.15, \mathrm{p}=0.04$ ) (Table 2). The odds ratio of photoreceptor defects in the chronic versus acute sun exposure groups was clinically significant at 9.84 $(95 \%$ CI $1.15-83.98, p=0.02)$ (Table 2$)$. 
Table 1. Baseline Demographics of Cases Reviewed

\begin{tabular}{|l|l|}
\hline \# of patients & 68 \\
\hline \# male & $41(60.3 \%)$ \\
\hline \# eyes & 101 \\
\hline laterality & 55 OD (54.5\%) \\
\hline mean age at examination & 39.5 years (range 10-86) \\
\hline mean time from exposure to exam & 1999 days (range 1-7300) \\
\hline \# greater than 1 year from exposure & $35(34.7 \%)$ \\
\hline mean BCVA & $20 / 61.5($ range 20/20-20/200) \\
\hline \# with fluorescein angiogram & $40(39.6 \%)$ \\
\hline \# with fluorescein angiogram defect & $20(50.0 \%)$ \\
\hline mean OCT central foveal thickness & 149.9 microns (range 85-237) \\
\hline \# receiving OCT & $101(100 \%)$ \\
\hline \# OCT vitreoretinal interface disruption & $1(1.0 \%)$ \\
\hline \# RPE disruption & $27(26.7 \%)$ \\
\hline \# inner high reflective layer disruption & $67(66.3 \%)$ \\
\hline \# outer photoreceptor segment disruption & $67(66.3 \%)$ \\
\hline \# inner photoreceptor segment disruption & $33(32.7 \%)$ \\
\hline $\begin{array}{l}\text { Baseline demographics and clinical characteristics of the patients from the literature } \\
\text { review were assessed. \# = number. OD = right eye. BCVA = best corrected visual } \\
\text { acuity. OCT = optical coherence tomography. RPE = retinal pigment epithelium. }\end{array}$ \\
\hline
\end{tabular}

Time from sun exposure to exam was analyzed in relation to RPE and photoreceptor defects of 80 eyes. Odds ratios are given for acute exposure $(<1$ year) as compared to chronic exposure ( $>1$ year). Photoreceptor defects were defined as lesions in the outer segments, inner segments, or inner-outer segment junction. P-value was calculated with Fisher's exact test. RPE = retinal pigment epithelium.

Analysis of each retinal layer defect with median BCVA revealed (Table 3): The median BCVA was 20/32 (logMAR 0.204 ) in the group with RPE defects and 20/40 (logMAR 0.301 ) in the group without RPE defects and the distributions were not significantly different $(\mathrm{p}=0.30)$ (Mann-Whitney U test). Eyes with inner high reflective layer defects had a median BCVA of 20/40 (logMAR 0.301) and eyes without had median BCVA of 20/45 (logMAR 0.352). Distributions between these two groups were not significantly different $(p$ $=0.28$ ) (Mann-Whitney U test). The median BCVA in eyes with outer segment defects was 20/40 (logMAR 0.301) and in eyes without was 20/30 (logMAR 0.176). The distributions did not significantly correlate with worse visual acuity $(p=0.15)$ (Mann-Whitney $U$ test). Median BCVA was 20/60 (logMAR 0.477) in eyes with inner segment defects and 20/30 (logMAR 0.176) in eyes without defects. Eyes with inner segment defects related to significantly worse visual acuity $(p<0.001)$ (Mann-Whitney $U$ test) (Table 3).

Age did not significantly correlate with better or worse visual acuity (Pearson's correlation coefficient $\mathrm{r}=0.1, \mathrm{p}=$ 0.34). Gender also did not correlate with better or worse visual acuity ( $\mathrm{p}=0.53$ ) (Mann-Whitney U test) (Table 4).

\section{DISCUSSION}

Acute solar retinopathy visual loss may resolve with time and patients may regain good visual acuity [7]. Although return of good visual acuity may occur, some patients continue to experience visual deficiencies such as a small persistent central or paracentral scotoma due to chronic solar damage to the photoreceptors and the RPE leading to retinal atrophy and degeneration $[3,8]$. Pathology may depend on the intensity, duration and light spectrum of solar exposure, ocular pigmentation, clarity of the ocular media and environmental conditions such as highly reflective surroundings and reduced atmospheric ozone [9].

Solar retinopathy was first described using time domain OCT by Bechmann and colleagues where they found a hyper-reflective area at the fovea and all retinal layers were affected [12]. Several studies using earlier modalities of OCT have shown transient increase in foveal reflectivity [12], reduced reflectivity from the retinal pigment epithelium [3,7-11] or disruption of the inner segment and outer segment of the photoreceptor layers [8-11,13-20]; but each

Table 2. Frequency and Odds Ratio of Time from Exposure and RPE and Photoreceptor Changes

\begin{tabular}{|c|c|c|c|c|c|}
\hline & \# of Eyes with & \# of Eyes without & Odds Ratio < $\mathbf{1}$ Year as Compared to $\geq \mathbf{1}$ Year & 95\% Confidence Interval & 1-Tailed p-Value \\
\hline \hline RPE defect & 23 & 57 & 2.78 & $1.08-7.15$ & 0.04 \\
\hline Photoreceptor defect & 67 & 13 & 0.10 & $0.01-0.87$ & 0.02 \\
\hline
\end{tabular}

\# Number.

Table 3. Frequency of OCT Findings and Association with BCVA

\begin{tabular}{|c|c|c|c|c|c|}
\hline OCT Findings & \# of Eyes with & \# of eyes Without & Median BCVA with & Median BCVA without & 1-Tailed p-Value \\
\hline \hline RPE defect & $27(26.7 \%)$ & $74(73.3 \%)$ & $20 / 32(0.204)$ & $20 / 40(0.301)$ & 0.30 \\
\hline Inner high reflective layer defect & $67(66.3 \%)$ & $34(33.7 \%)$ & $20 / 40(0.301)$ & $20 / 45(0.352)$ & 0.28 \\
\hline Outer segment defect & $67(66.3 \%)$ & $34(33.7 \%)$ & $20 / 40(0.301)$ & $20 / 30(0.176)$ & 0.15 \\
\hline Inner segment defect & $33(32.7 \%)$ & $68(67.3 \%)$ & $20 / 60(0.477)$ & $20 / 30(0.176)$ & $<\mathbf{0 . 0 0 1}$ \\
\hline
\end{tabular}

The association between OCT findings and BCVA were assessed. P-values were calculated using the Mann-Whitney U test. Bold font denote statistical significance with $\mathrm{p} \leq 0.05$. $\mathrm{OCT}=$ optical coherence tomography. $\#=$ number. $\mathrm{RPE}=$ retinal pigment epithelium. BCVA = best corrected visual acuity in Snellen (logMAR in parentheses). 
case series demonstrated that OCT retinal structural alterations may not be consistent in every case of solar retinopathy [2-4,7-20]. Acute changes seen on OCT from solar retinopathy have been described to affect predominantly the outer photoreceptor segments and may even resolve with time $[3,4]$. Patients with a chronic history (at least 1 year or more) of solar retinopathy exposure primarily have disruption between the inner and outer segments of photoreceptors [4].

Table 4. Relationship Between Gender and BCVA

\begin{tabular}{|c|c|c|c|}
\hline & \# of Eyes & Median BCVA & 2-Tailed p-Value \\
\hline \hline Male & 63 & $20 / 32(0.204)$ & ---- \\
\hline Female & 38 & $20 / 45(0.352)$ & 0.53 \\
\hline
\end{tabular}

Analysis was performed on gender in relationship to BCVA. P-value was calculated using the Mann-Whitney U test. \# = number. BCVA = best corrected visual acuity in Snellen (logMAR in parentheses).

In the present study, utilizing the high definition imaging from the Spectralis SD-OCT (Heidelberg Engineering, Vista, California, USA), solar retinopathy was shown to specifically damage the inner and outer segment of the photoreceptors in both acute (Cases 1 and 2) and chronic (Case 3) presentations, without involvement of the RPE. In the literature review, RPE defects were more prominent in the acute exposure group based on the odds ratio $(p=0.04)$ and photoreceptor defects were found more often in the chronic exposure group $(\mathrm{p}=0.02)$. While this was significant, RPE defects were still observed in the chronic exposure group and photoreceptor defects were also seen in the acute exposure group. Losses at the RPE layer may represent photochemical damage to the pigmented RPE layer $[5,7,8]$, and acute disruption of the outer segment layer from solar exposure may be secondary to loss of RPE function therefore indirectly affecting the photoreceptor segments [4].

Not only are these OCT changes correlated with time from exposure to exam, time interval also affects visual acuity. Less than one year from exposure to examination showed better visual acuity when compared to more than one year from exposure $(\mathrm{p}<0.001)$. It is possible that acute sun exposure causes damage to both the RPE and photoreceptor layers, and in chronic cases leads to permanent disruption of the inner and outer segments with photoreceptor cells demonstrating vesiculation and fragmentation of the photoreceptor lamellae [6]. Presence of optically empty spaces or full-thickness disruption of the whole photoreceptor band just beneath the fovea may explain permanent visual loss, whereas involvement outside of the fovea does not affect vision $[13,14]$.

Based on our case series and literature review, our results suggest that the photoreceptor layer may be the layer most likely to be permanently damaged by solar retinopathy and lead to overall worse long-term vision. Lesions in the RPE layer, inner high reflective layer and outer photoreceptor segment layer did not affect best corrected visual acuity. Only the inner photoreceptor lesions were significantly related to worse visual acuity $(\mathrm{p}<0.001)$. One explanation for this is that the high resolution Spectralis SD-OCT allowed for better characterization of the retinal layers affected compared to previous reports. More data gathered by Spectralis SD-OCT will continue to help address this issue. Of note, several papers from the literature review used a lower resolution time domain OCT, and error may have been introduced when defining the location of the lesions.

In conclusion, this present series of patients utilizing high definition SD-OCT imaging suggests that solar retinopathy OCT findings may occur primarily at the inner photoreceptor segment layers leading to foveal atrophy. Acute changes defined as less than one year from exposure to exam tend to affect vision less than more chronic photoreceptor changes in the inner photoreceptor layer or at the junction of the inner and outer photoreceptor layers. Limitation of our study includes the number of eyes evaluated but with continued use of SD-OCT, future studies can utilize this modality of OCT imaging to analyze larger numbers of patients with solar retinopathy and elucidate possible causes of visual loss and supplement clinical exams.

\section{ACKNOWLEDGEMENT}

Supported by a Grant from Research to Prevent Blindness (RPB).

\section{CONFLICT OF INTEREST}

\author{
Declared none.
}

\section{REFERENCES}

[1] Gass JDM. Stereoscopic atlas of macular diseases: diagnosis and treatment. Vol. $4^{\text {th }}$. St. Louis: Mosby 1997; pp. 760-3.

[2] Chen RW, Gorczynska I, Srinivasan VJ, et al. High-speed ultrahigh-resolution optical coherence tomography findings in chronic solar retinopathy. Retin Cases Brief Rep 2008; 2(2):103-5.

[3] Codenotti M, Patelli M, Brancato R. OCT Findings in patients with retinopathy after watching a solar eclipse. Ophthalmologica 2002; 216(6): 463-6.

[4] Jain A, Desai RU, Charalel RA, et al. Solar retinopathy comparison of optical coherence tomography (OCT) and fluorescein angiography (FA). Retina 2009; 29(9):1340-5.

[5] Tso MO, La Piana FG. The human fovea after sungazing. Trans Am Acad Ophthalmol Otolaryngol 1975; 79(6): 788-95.

[6] Hope-Ross MW, Mahon GJ, Gardiner TA, Archer DB. Ultrastructural findings in solar retinopathy. Eye 1993; 7(Pt 1): 2933.

[7] Chen JC, Lee LR. Solar retinopathy and associated optical coherence tomography findings. Clin Exp Optom 2004; 87(6): 3903 .

[8] Garg SJ, Martidis A, Nelson ML, Sivalingam A. Optical coherence tomography of chronic solar retinopathy. Am J Ophthalmol 2004; 137(2): 351-4.

[9] Stangos AN, Petropoulos IK, Pournaras JC, et al. Optical coherence tomography and multifocal electroretinogram findings in chronic solar retinopathy. Am J Ophthalmol 2007; 144(1):131-4.

[10] Steinkamp PN, Watzke RC, Solomon JD, Portland O. An unusual case of solar retinopathy. Arch Ophthalmol 2003; 121(12):1798-9.

[11] Kaushik S, Gupta V, Gupta A. Optical coherence tomography findings in solar retinopathy. Ophthalmic Surg Lasers Imaging 2004; 35(1): 52-5.

[12] Bechmann M, Ehrt O, Thiel MJ, et al. Optical coherence tomography findings in early solar retinopathy. Br J Ophthalmol 2000; 84(5): 547-8.

[13] Jorge R, Costa RA, Quirino LS, et al. Optical coherence tomography findings in patients with late solar retinopathy. Am J Ophthalmol 2004; 137(6): 1139-42.

[14] Gulkilik G, Taskapili M, Kocabora S, et al. Association between visual acuity loss and optical coherence tomography findings in patients with late solar retinopathy. Retina 2009; 29(2): 257-61.

[15] Calvo-Gonzalez C, Reche-Frutos J, Santos-Bueso E, et al. Optical coherence tomography in solar eclipse retinopathy. Arch Soc Esp Oftalmol 2006; 81(5): 297-300. 
[16] Kung Y, Wu T, Sheu S. Subtle solar retinopathy detected by fourier-domain optical coherence tomography. J Chin Med Assoc 2010; 73(7): 396-8.

[17] Devadason DS, Mahmood S, Stanga PE. Solar retinopathy in a patient with bipolar affective disorder. Br J Ophthalmol 2006; 90(2): 247.

[18] Macarez R, Vanimschoot M, Ocamica P, Kovalski JL. Optical coherence tomography follow-up of a case of solar maculopathy. J Fr Ophtalmol 2007; 30(3): 276-80.

[19] Issa PC, Fleckenstein M, Scholl HP, Holz FG, Meyer CH. Confocal scanning laser ophthalmoscopy findings in chronic solar retinopathy. Ophthalmic Surg Lasers Imaging 2008; 39(6): 497-9.
[20] Comander J, Gardiner M, Loewenstein J. High-resolution optical coherence tomography findings in solar maculopathy and the differential diagnosis of outer retinal holes. Am J Ophthalmol 2011; 152(3): 413-9.

[21] Cho HJ, Yoo ES, Kim CG, Kim JW. Comparison of spectraldomain and time-domain optical coherence tomography in solar retinopathy. Korean J Ophthalmol 2011; 25(4): 278-81.

[22] Bonyadi MH, Soheilian R, Soheilian M, Peyman GA. Spectraldomain optical coherence tomography features of mild and severe acute solar retinopathy. Ophthalmic Surg Lasers Imaging 2011; 42(5): e84-6.

(C) Chen et al.; Licensee Bentham Open.

This is an open access article licensed under the terms of the Creative Commons Attribution Non-Commercial License (http://creativecommons.org/licenses/by$\mathrm{nc} / 3.0 /$ ) which permits unrestricted, non-commercial use, distribution and reproduction in any medium, provided the work is properly cited. 